\title{
Characteristics of Symmetric Left-Handed Material Slab Waveguide
}

\author{
Khitam Y. Elwasife ${ }^{1}$, Sofyan A. Taya ${ }^{2}$ \\ ${ }^{1,2}$ Physics Department, Islamic University of Gaza, Gaza, Palestine.
}

\begin{abstract}
In this paper, a symmetric three-layer slab waveguide is investigated. The guiding layer and the surrounding medium are assumed to be left-handed materials of negative permittivity and permeability. The dispersion equations are derived for both TE and TM polarizations. The properties of the waveguide structure are investigated for different mode orders and for different parameters of the left-handed materials. The field profiles of the guided modes are presented in order to understand these properties.
\end{abstract}

Keywords - Slab waveguide, field profile, left-handed materials.

\section{Introduction}

Left-handed materials (LHMs) have received an increasing interest because of their potential applications in future optical technology [1-12]. Veselago predicted a number of remarkable properties of electromagnetic waves propagating in a material with simultaneously negative permittivity $\varepsilon$ and permeability $\mu$ [10]. The symmetric and asymmetric three-layer LHM slab waveguide structures were analyzed and some properties of the guided modes were reported [13-16]. Typical properties of these waveguides including the absence of the fundamental mode and backward propagating waves with negative power flux were found. Waveguide structures comprising a layer made of LHM have unique properties and have become an interesting topic for researchers in physics and engineering. Hundreds of papers have been published to investigate the dispersion properties of LHM waveguide structures. We give here some important examples of these studies. The characteristics of guided waves through a slab waveguide of uniaxially anisotropic dispersive LHM were investigated [17]. The results indicate that the mode properties are closely dependent on the frequency. Fourand five-layer waveguide structures including one LHM layer were proposed and the propagation of TE and TM-polarized waves in such structures has been investigated $[13,18,19]$. In these structures, the effect of the negative parameters of the LHM on the propagation characteristics was examined. The theory of LHMs and their electromagnetic properties, possible future applications, physical remarks, and intuitive justifications were provided by Engheta [20]. Guided modes in an asymmetric waveguide structure with LHM layer surrounded by air and metal were presented [21-22]. A graphical method was proposed to determine the guided modes in the structure proposed in Ref. 21. Guided modes propagating along a dielectric slab waveguide with both double negative and single negative materials were investigated [23]. Three normalized parameters were used to study the dispersion characteristics of different asymmetric slab waveguide structures comprising LHM layer [14,24]. LHMs have been studied for many applications such as optical waveguide sensing applications [25-38], cloaking [39], micro strip patch antenna [40], and wave absorbers [41].

In this study, a symmetric three-layer slab waveguide structure is considered. All media are assumed to be LHMs. To the best of our knowledge, this structure hasn't been investigated in the literature. Both TE and TM polarizations are treated. The properties and field profiles of the proposed structure are presented in details for different mode orders and different parameters of the media constituting the waveguide.

\section{Theory}

We assume a symmetric three-layer slab waveguide structure as shown in Fig. 1. The guiding layer is made of a LHM and it occupies the region $0<\mathrm{z}<\mathrm{d}$. The cladding and substrate are also assumed to be identical LHMs. The cladding, guiding layer, and substrate have the parameters $\left(\varepsilon_{\mathrm{c}}, \mu_{\mathrm{c}}\right),\left(\varepsilon_{\mathrm{f}}, \mu_{\mathrm{f}}\right)$, and $\left(\varepsilon_{\mathrm{c}}, \mu_{\mathrm{c}}\right)$, respectively. The parameters $\varepsilon_{j}$ and $\mu_{j}$ represent the relative permittivity and permeability of the layer. We first consider TE modes in which the following field components exist: $E_{y}, H_{x}$, and $H_{z}$. 


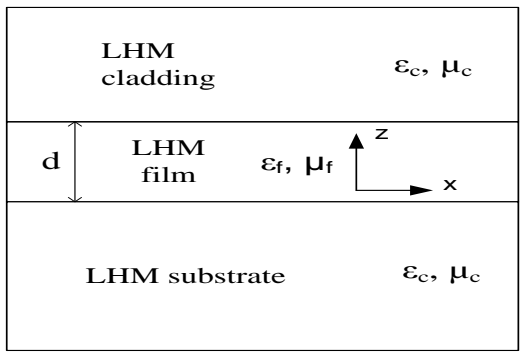

Fig. 1. Symmetric three-layer waveguide structure with all media are made of LHMs.

Helmholtz equation is given by

$\frac{\partial^{2} E_{y}}{\partial z^{2}}+\left(\omega^{2} \mu_{i} \varepsilon_{i}-\beta^{2}\right) E_{y}=0$

where $\beta$ is the propagation constant and $i=\mathrm{c}, f$, or $\mathrm{s}$ indicate cladding, film or substrate, respectively. For oscillating guided TE modes, the electric field is polarized along the y-axis and can be expressed as

$\left.\begin{array}{l}E_{y}=E_{c} \exp \left[-\alpha_{c}(z-d)\right] e^{i \beta x}, z>d \\ E_{y}=E_{f} \cos \left[k_{f} z-\varphi_{s}\right] e^{i \beta x}, 0<z<d \\ E_{y}=E_{s} \exp \left[\alpha_{c} z\right] e^{i \beta x}, z<0\end{array}\right\}$,

where $\alpha_{c}^{2}=\beta^{2}-k^{2} \varepsilon_{c} \mu_{c}, k_{f}^{2}=k^{2} \varepsilon_{f} \mu_{f}-\beta^{2}, k$ is the free space wave number, $E_{c}, E_{f}, E_{s}$ are field amplitudes in the cladding, film, and substrate, respectively and $\varphi_{s}$ is a phase shift.

The magnetic field components $\left(H_{x}\right.$ and $\left.H_{z}\right)$ of the TE modes can be calculated using $H_{z}=\frac{-k_{0} N}{\omega \mu} E_{y}$ and $H_{x}=\frac{i}{\omega \mu} \frac{\partial E_{y}}{\partial z}$, where $N=\beta / k$ is the effective modal index.

The magnetic field components are found to be

$$
H_{z}=\left\{\begin{array}{l}
-\frac{k_{o} N}{\omega \mu_{o} \mu_{c}} E_{c} \exp \left[-\alpha_{c}(z-d)\right] e^{i \beta x}, z>d \\
-\frac{k_{o} N}{\omega \mu_{o} \mu_{f}} E_{f} \cos \left[k_{f} z-\varphi_{s}\right] e^{i \beta x}, 0<z<d \\
-\frac{k_{o} N}{\omega \mu_{o} \mu_{c}} E_{c} \exp \left[\alpha_{c} z\right] e^{i \beta x}, z<0
\end{array}\right.
$$

and

$$
H_{x}=\left\{\begin{array}{l}
\frac{-i \alpha_{c}}{\omega \mu_{o} \mu_{c}} E_{c} \exp \left[-\alpha_{c}(z-d)\right] e^{i \beta x}, z>d \\
\frac{-i k_{f}}{\omega \mu_{o} \mu_{f}} E_{f} \sin \left[k_{f} z-\varphi_{s}\right] e^{i \beta x}, 0<z<d \\
\frac{i \alpha_{c}}{\omega \mu_{o} \mu_{c}} E_{s} \exp \left[\alpha_{c} z\right] e^{i \beta x}, z<0
\end{array}\right.
$$

Applying the boundary conditions such that $E_{y}$ and $H_{x}$ are continuous at $\mathrm{z}=0$ and $\mathrm{z}=\mathrm{d}$, we obtain 


$$
E_{f}=\frac{E_{s}}{\sqrt{\frac{1}{1+\frac{\alpha_{c}^{2} \mu_{f}^{2}}{k_{f}^{2} \mu_{c}^{2}}}}},
$$

$E_{c}=E_{s}\left[\cos \left(k_{f} d\right)+\sin \left(k_{f} d\right) \frac{\alpha_{c}}{k_{f}} \frac{\mu_{f}}{\mu_{c}}\right]$,

$\varphi_{s}=\tan ^{-1}\left(\frac{\alpha_{c}}{k_{f}} \frac{\mu_{f}}{\mu_{c}}\right)$.

The dispersion relation of the guided modes can be obtained as follow

$\tan \left(k_{f} d\right)=\frac{2\left(\frac{\mu_{f} \alpha_{c}}{\mu_{c} k_{f}}\right)}{1-\left(\frac{\mu_{f}^{2} \alpha_{c}^{2}}{\mu_{c}^{2} k_{f}^{2}}\right)}$,

which usually written as

$k_{f} d-2 \tan ^{-1}\left(\frac{\mu_{f} \alpha_{c}}{\mu_{c} k_{f}}\right)-m_{1} \pi=0$.

where $m_{1}=0,1,2, \ldots$ is the mode order.

For TM polarization, the dispersion relation is similar to that of TE case with $\varepsilon_{i}$ replaces $\mu_{i}$, it takes the form

$k_{f} d-2 \tan ^{-1}\left(\frac{\varepsilon_{f} \alpha_{c}}{\varepsilon_{c} k_{f}}\right)-m_{1} \pi=0$.

\section{Numerical Results and Discussion}

In the present work, we treat three-layer symmetric waveguide structure in which all media are LHMs such that $\varepsilon_{c}, \varepsilon_{f}, \mu_{c}$ and $\mu_{f}$ have the forms $\varepsilon_{j}=\varepsilon_{r}+i \varepsilon_{i}$ and $\mu_{j}=\mu_{r}+i \mu_{i}(j=c, f)$ with $\varepsilon_{r}$ and $\mu_{r}$ are negative. In the analysis below we have assumed a wavelength of $1550 \mathrm{~nm}$. Equation (9) was solved numerically for the propagation constant. In Fig. 2, the propagation constant $\beta$ versus the guiding layer thickness $d$ is illustrated for different TE modes for symmetric waveguide having $\mu \mathrm{c}=\mu \mathrm{f}=-7.5+0.001 \mathrm{i}, \varepsilon \mathrm{c}=-3.7+0.001 \mathrm{i}$, and $\varepsilon \mathrm{f}=-$ $5.7+0.001 \mathrm{i}$. As the figure reveals, the propagation constant of the guided mode is enhanced with increasing the thickness of the guiding layer as well as the mode order. Both of these behaviors are well known in the propagation of electromagnetic waves in slab waveguides. As the thickness of the guiding layer increases, the propagating wave confinement increases and hence the propagation constant as well as the effective index of the guided mode are enhanced. The same effect is observed as the mode order increases from the fundamental mode $(\mathrm{m} 1=0)$ to higher modes. The existence of the fundamental mode is one of the most important features that can be seen in Fig. 2. It was reported that the fundamental mode does not exist in waveguide configurations having a LHM guiding layer surrounded by two dielectric media [4]. The propagation constant of TE0 guided mode of the proposed structure is plotted as a function of the guiding layer thickness for different values of the guiding film permittivity in Fig. 3. In all figures, we plot the real part of the propagation constant. As can be seen from the figure, the propagation constant increases as the absolute value of the real part of the guiding layer permittivity increases from 5.7 to 5.9. This increase in the propagation constant means that the confinement of the wave in the guiding layer is enhanced and the evanescent fields in the surroundings diminish.

The modal propagation constant of a certain mode is essentially dependent on the thickness and optical parameters of the guiding layer. Figure 4 illustrates the properties of TE0 guided mode of the proposed structure for different values of the guiding film permeability. The effect of increasing the permeability of the guiding 
layer is similar to that of increasing its permittivity. The increase in any of them enhances the refractive index of the guiding layer which in turn enhances the propagation constant.

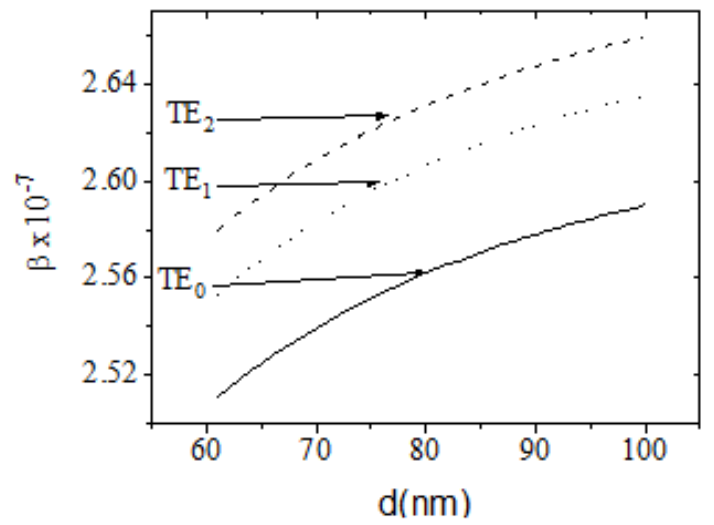

Fig. 2. Propagation constant of the proposed structure versus thickness of the guiding layer for different TE modes for $\lambda=1550 \mathrm{~nm}, \mu_{c}=\mu_{f}=-7.5+0.001 \mathrm{i}, \varepsilon_{c}=-3.7+0.001 \mathrm{i}$, and $\varepsilon_{f}=-5.7+0.001 \mathrm{i}$.

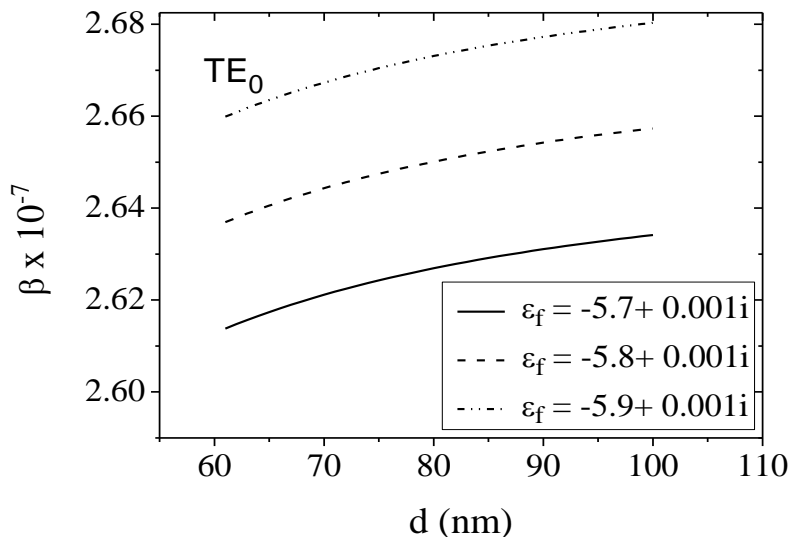

Fig. 3. Propagation constant of the proposed structure versus thickness of the guiding layer for different values of the guiding layer permittivity for $\lambda=1550 \mathrm{~nm}, \mu_{c}=\mu_{f}=-7.5+0.001 \mathrm{i}$, and $\varepsilon_{c}=-3.7+0.001 \mathrm{i}$.

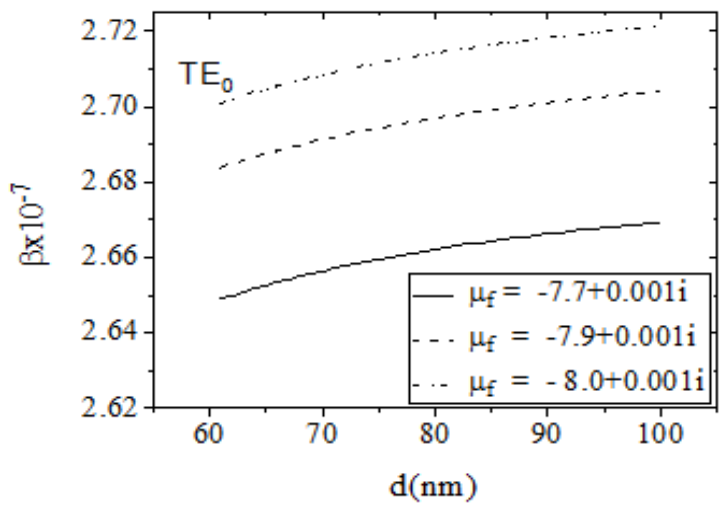

Fig. 4. Propagation constant of the proposed structure versus thickness of the guiding layer for different values of the guiding layer permeability for $\lambda=1550 \mathrm{~nm}, \mu_{c}=-7.5+0.001 \mathrm{i}, \varepsilon_{c}=-3.7+0.001 \mathrm{i}$, and $\varepsilon_{f}=-5.7+0.001 \mathrm{i}$.

In Figs. 5 and 6, we investigate the effect of the surrounding medium parameters on the properties of the structure. Figure 5 shows a slight effect of the permittivity of the surrounding medium on the propagation constant. As the absolute value of the real part of the permittivity increases, a rarely detectable enhancement in the propagation constant is observed. The behavior of the propagation constant with the permeability of the surrounding medium is completely different. As Fig. 6 reveals, a little enhancement in the propagation constant of the proposed structure is observed when the permeability of the surrounding medium decreases. 
It is significant to examine the shape of the corresponding field components. The transverse profiles of $\mathrm{TE}_{0}, \mathrm{TE}_{1}$ and $\mathrm{TE}_{2}$ guided modes are plotted in Fig.7. As can be seen from the figure, the electric field has the usual configuration. It oscillates in the guiding layer and evanescent in the surrounding media. The two peaks of $\mathrm{TE}_{2}$ guided mode are symmetric due to the symmetry of the structure. For the same reason, the field distributions of all mode orders are observed to be identical in the cladding and substrate. As mentioned above, the fundamental mode is found to exist in the proposed structure in contrary to waveguide structures comprising a LHM surrounded by dielectric media. To explain the behavior observed in Fig. 3 and Fig. 4, we plot the field profile for the fundamental mode for different values of the guiding layer permittivity in Fig. 8 and for different values of the guiding layer permeability in Fig. 9. The enhancement of the evanescent field with decreasing the absolute values of $\varepsilon_{f}$ and $\mu_{f}$ is clear in Fig. 8 and Fig. 9.

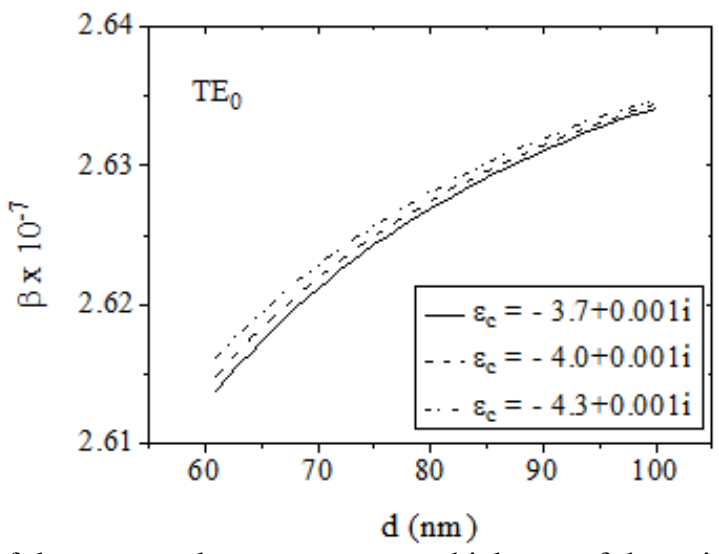

Fig. 5. Propagation constant of the proposed structure versus thickness of the guiding layer for different values of the surrounding medium permittivity for $\lambda=1550 \mathrm{~nm}, \mu_{c}=\mu_{f}=-7.5+0.001 \mathrm{i}$, and $\varepsilon_{f}=-5.7+0.001 \mathrm{i}$.

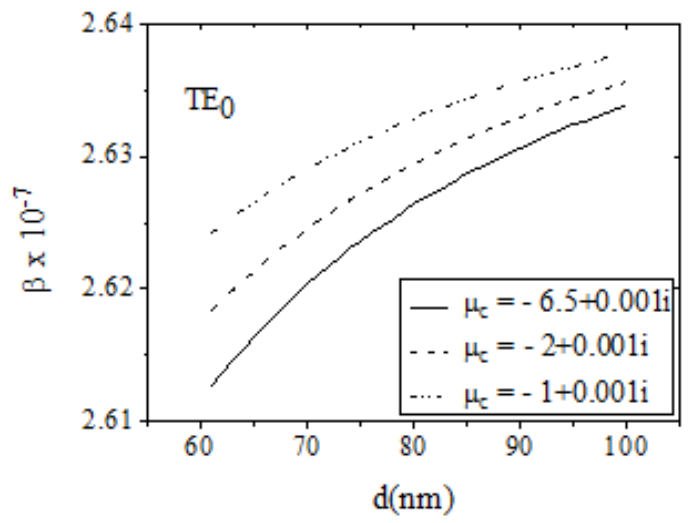

Fig. 6. Propagation constant of the proposed structure versus thickness of the guiding layer for different values of the surrounding medium permeability for $\lambda=1550 \mathrm{~nm}, \mu_{f}=-7.5+0.001 \mathrm{i}, \varepsilon_{c}=-3.7+0.001 \mathrm{i}$, and $\varepsilon_{f}=-$

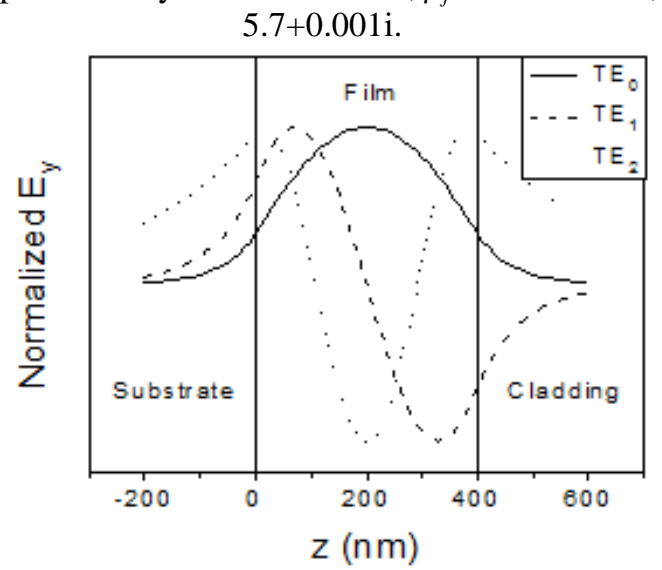

Fig. 7. Transverse profiles of the guided modes for different TE modes for $\lambda=1550 \mathrm{~nm}, \mu_{c}=\mu_{f}=-7.5+0.001 \mathrm{i}$, $\varepsilon_{c}=-3.7+0.001 \mathrm{i}$, and $\varepsilon_{f}=-5.7+0.001 \mathrm{i}$. 
We now investigate the properties of the proposed structure when the surrounding medium is righthanded material (RHM) with positive index of refraction. Figure 10 shows the propagation constant versus the thickness of the guiding layer when the surrounding medium is assumed to be RHM and when it is a LHM. The two curves are identical in shape with a little enhancement in the propagation constant when the surrounding medium is RHM. This means that the presence of LHM as a surrounding medium allows a stronger evanescent field to extend from the guiding layer to the surrounding medium. Therefore, the structure with LHM surrounding medium is a better candidate for applications requiring the strengthening of the evanescent field such as optical waveguide sensing [42-44]. The principle of operation of waveguide sensors is based on the evanescent field in the cladding layer in which the material to be detected (analyte) exists. The guided wave in a waveguide structure senses an effective refractive index of the guided mode that depends on the thickness of the guiding layer and the refractive indices of all media constituting the waveguide structure. As a result, any change in the refractive index of the analyte in the cladding layer results in a change in the effective refractive index of the guided mode. The basic sensing principle of optical waveguide sensors is to measure the changes in the effective index due to changes in the index of the analyte. It is worth mentioning that Fig. 10 shows the first guided mode $(\mathrm{m}=1)$ since the fundamental mode does not exist in the case of a LHM guiding layer surrounded by RHMs.

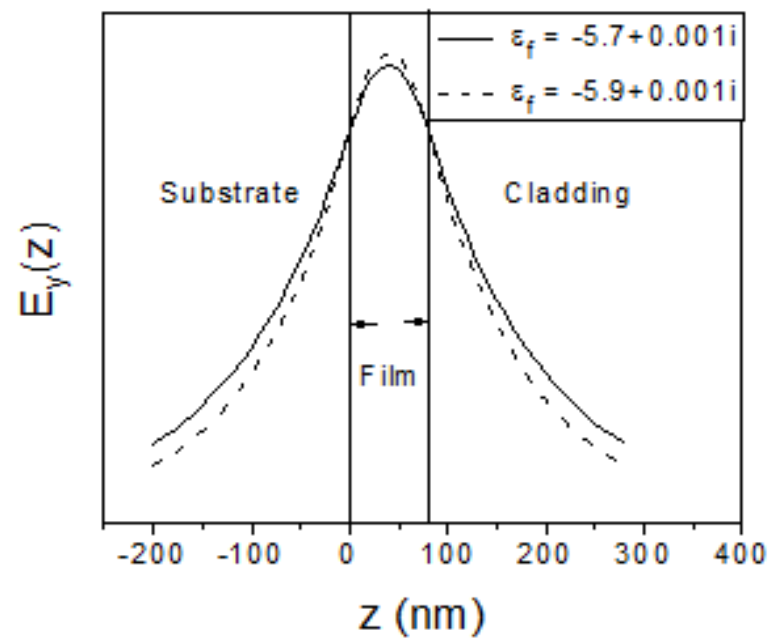

Fig. 8. Transverse profiles of the fundamental mode for different values of the guiding layer permittivity for $\lambda=$ $1550 \mathrm{~nm}, \mu_{c}=\mu_{f}=-7.5+0.001 \mathrm{i}$, and $\varepsilon_{c}=-3.7+0.001 \mathrm{i}$.

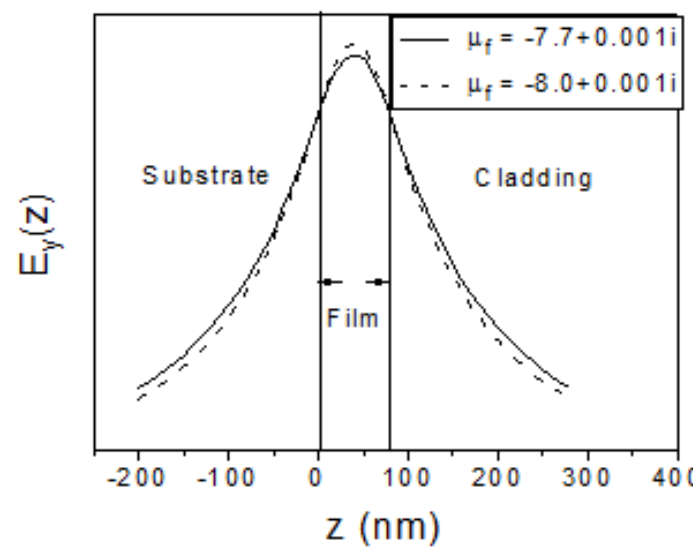

Fig. 9. Transverse profiles of the fundamental mode for different values of the guiding layer permeability for $\lambda$ $=1550 \mathrm{~nm}, \mu_{c}=-7.5+0.001 \mathrm{i}, \varepsilon_{c}=-3.7+0.001 \mathrm{i}$, and and $\varepsilon_{f}=-5.7+0.001 \mathrm{i}$. 


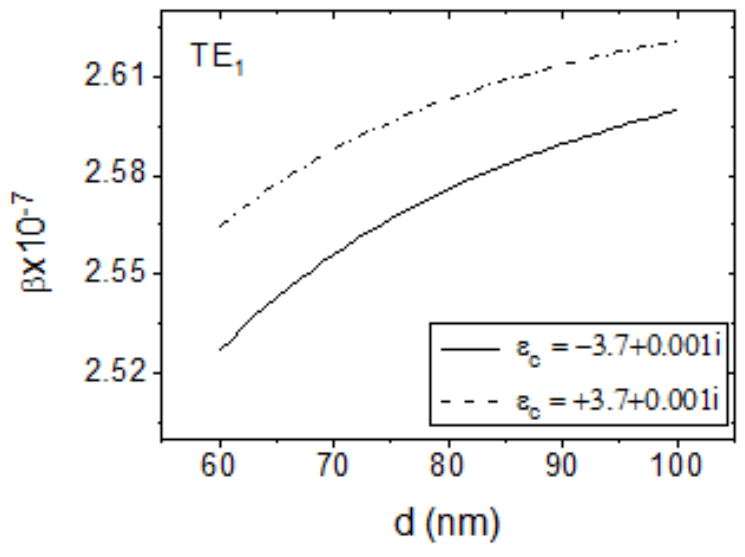

Fig. 10. Propagation constant of the proposed structure versus thickness of the guiding layer when the surrounding medium is LHM and RHM for $\lambda=1550 \mathrm{~nm}, \mu_{f}=-7.5+0.001 \mathrm{i}$, and $\varepsilon_{f}=-5.7+0.001 \mathrm{i}$.

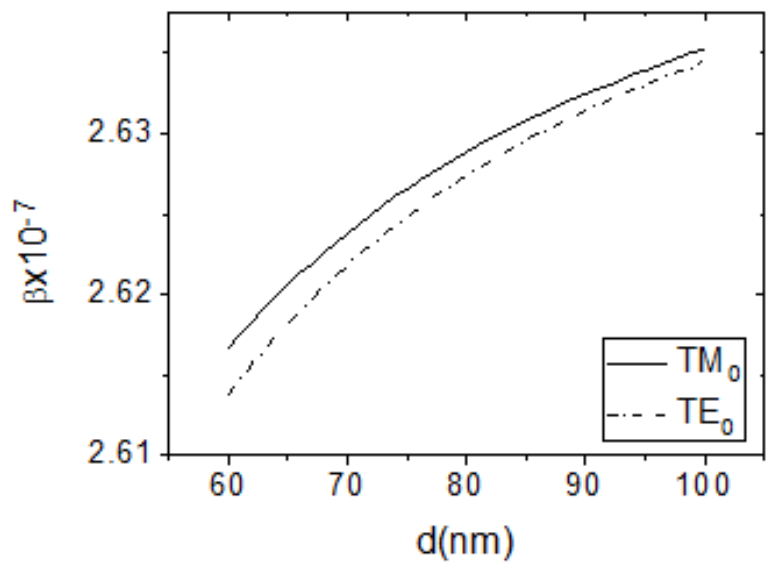

Fig. 11. Comparison between the fundamental modes $\mathrm{TE}_{0}$ and $\mathrm{TM}_{0}$ for $\lambda=1550 \mathrm{~nm}, \mu_{c}=\mu_{f}=-7.5+0.001 \mathrm{i}, \varepsilon_{c}=$ $-3.7+0.001 \mathrm{i}$, and $\varepsilon_{f}=-5.7+0.001 \mathrm{i}$.

We finally investigate the p-polarized (TM) case. As mentioned above, the dispersion relations of both polarizations are similar with $\varepsilon_{i}$ replaces $\mu_{i}$ for p-polarized light. The properties of the fundamental mode of both polarizations are illustrated in Fig. 11. The two curves are very close to each other with a slight enhancement in the propagation constant for p-polarization.

\section{Conclusions}

In this work, we analyzed the properties of TE and TM modes in a symmetric three-layer slab waveguide structure. The core layer, cladding and substrate were assumed to be left- handed materials. The mathematical derivation of the dispersion relations was presented. The behavior of the propagation constant with the guiding layer thickness was investigated for different mode orders and different parameters of the lefthanded materials. The field profiles of the guided modes are presented in order to understand these properties. The electric field distribution in the layers of the structure was found to have the usual configuration. It oscillates in the guiding layer and evanescent in the surrounding media.

\section{References}

[1] R.A. Shelby, D.R. Smith, and S. Schultz, Experimental verification of a negative index of refraction, Science, 292(6), $2001,77-79$.

[2] J.B. Pendry, S.A. Ramarkrishna, Focusing light using negative refraction, Phys., J. Condens. Matter, 15(37), 2003, 6345-6364.

[3] H. Wang, Z. Y. Xiao, S.P. Li, Guided modes in slab waveguides with a left handed material cover or substrate, Opt. commun. J., 281(4), 2008, 607-613.

[4] D.R. Smith, W.J. Padilla, D.C. Vier, S.C. Nemat Nasser and S. Schultz, Composite Medium with Simultaneously Negative Permeability and Permittivity , Phys. Rev. Lett., 84(18), 2000, 41-84.

[5] Ruppin, Surface polaritons and extinction properties of a left-handed material cylinder, Phys. Condens. Matter , 13(34), 2001, 1811-1819.

[6] Kh. Y. Elwasife and S. A. Taya, Guided waves in a left-handed material guiding film with a ferrite cladding, Instasci Journal of Physics, 1(1), 2011, 21-31.

[7] S. A. Taya, H. M. Kullab, and I. M. Qadoura, Dispersion properties of slab waveguides with double negative material guiding layer and nonlinear substrate, Journal of the optical society of America B, J. Opt. Soc. Am. B, 3(7) , 2013, $2008-2013$.

[8] S. A. Taya, Kh.Y. Elwasife, and H. M. Kullab, Dispersion properties of anisotropic-metamaterial slab waveguide structure, Optica Applicata, 43(4), 2013, 857-869. 
[9] M. Abadla and S. A. Taya, Excitation of TE surface polaritons in different structures comprising a left-handed material and a metal, Optik-International Journal for Light and Electron Optics, Optik - Int. J. Light Electron Opt., 125(3), 2014, $1401-1405$.

[10] V.G. Veselago, The electrodynamics of substances with simultaneously negative values of $\varepsilon$ and $\mu$, Sov. Phy. Usp., 10(4), 1968, 509-514.

[11] H.M. Kullab, S.A. Taya, and T. M. El-Agez, Metal-clad waveguide sensor using a left-handed material as a core layer, J. Opt. Soc. Am. B, 29(5), 2012, 959-964.

[12] I.V. Shadrivov, A.A. Sukhorukov, and Y.S. Kivshar, Guided modes in negative-refrative-index waveguides , Phys. Review E, 67(5), 2003, 057602-057610.

[13] S.A. Taya, E.J. El-Farram, and M.M. Abadla, Symmetric multilayer slab waveguide structure with a negative index material: TM case, Optik, J., 123(24), 2012, 2264-2268.

[14] S.A. Taya and I.M. Qadoura, Guided modes in slab waveguides with negative index cladding and substrate, Optik, J. 124(4), 2013, $1431-1436$.

[15] B.L. Wu, T.M. Grzegorczyk, Y. Zhang, and J.A. Kong, Guided modes with imaginary transverse wave number in a slab waveguide with negative permittivity and permeability. J. Appl. Phys., 93(13), 2003, 9386.

[16] H. Cory and A. Barger, Surface-wave propagation along a metamaterial slab, Microwave and optical technology letters, 38(5), 2003, 392-395.

[17] S.H. Liu, C.H. Liang, W. Ding, L. Chen, and W.T. Pan, Electromagnetic Wave Propagation Through a Slab Waveguide of Uniaxally Anisotropic Dispersive Metamaterial. Progress In Electromagnetics Research, PIER. 76(2), $2007,467-475$.

[18] L.F. Shen and H. Wang, S.P. Li, Propagation Properties for Five-Layer Symmetric Slab Waveguides including Left-Handed Materials, Optoelectronics Letters, 4(3), 2008, 165-167.

[19] M.M. Abadla and S.A. Taya, Characteristics of left-handed multilayer slab waveguide structure, The Islamic University Journal of Series of Natural Studies and Engineering, 10(9), 2011, 19:57-70.

[20] N. Engheta, Metamaterials with negative permittivity and permeability background, salient features, and new trends, IEEE MTT_S Int. Microwave Symp. Digest, 1(3), 2003, 187-194.

[21] S.A. Taya and Kh.Y. Elwasife, Guided modes in a metal-clad waveguide comprising a left-handed material as a guiding layer, International Journal of Research and Reviews in Applied Science, 13(1), 2012, 294-305.

[22] Y. He, X. Zhang, Y. Yang, and Ch. Li, Guided modes in asymmetric metal-cladding left-handed material waveguides, Chinese Optics Lett., 5(9), 2011, 052301.

[23] P. Dong, H. Wei Yang, Guided modes in slab waveguides with both double-negative and single-negative materials, Optica Applicata,J. 40(4), 2010, 873-882.

[24] I. M. Qadoura, S. A. Taya, and Kh.Y. El-wasife, Scaling rules for a slab waveguide structure comprising nonlinear and negative index materials, International Journal of Microwave and Optical Technology (IJMOT), 7(5), 2012, 349-357.

[25] H. M. Kullab and S. A. Taya, Peak type metal-clad waveguide sensor using negative index materials, International Journal of Electronics and Communications. Int. J. Electron. Commun. (AEÜ), 67(11), 2013, 905-992.

[26] H. M. Kullab and. A. Taya, Transverse magnetic peak type metal-clad optical waveguide sensor, Optik - Int. J. Light Electron Opt. 145(1), 2014, 97-100.

[27] S. A. Taya and H. M. Kullab, Optimization of transverse electric peak type metal-clad waveguide sensor using double negative materials, Applied Physics A, (in press).

[28] S. A. Taya, Slab waveguide with a.ir core layer and anisotropic left-handed material claddings as a sensor, Opto-Electron. Rev., 22(4), 2014, 252-257.

[29] T. Koschny .,R. Moussa, C. Soukoulis, Limits on the amplification of evanescent waves of left-handed materials, J. Opt. Soc. Am. B. 23(3), 2006, 485-489.

[30] S. A. Taya, P-polarized surface waves in a slab waveguide with left-handed material for sensing applications, J. Magn. Magn. Mater., 377(3), 2015, 281-285.

[31] A. Upadhyay, Y. Prajapati Singh V., J.P.Saini, Comprehensive study of reverse index waveguide based sensor with metamaterials as a guiding layer, Optics Communications 348, 2015, 71-76.

[32] S. A. Taya, Theoretical investigation of slab waveguide sensor using anisotropic metamaterials", Optica Appl., 45(3), 2015, 405417 .

[33] [33] H. M. Kullab, I. M. Qadoura, and S. A. Taya, Slab waveguide sensor with left-handed material core layer for detection an adlayer thickness and index, J. Nano-Electron. Phys., 7(2), 2015, 20- 39.

[34] A. Upadhyay, Y.K. Prajapati , V. Singh., J.P. Saini, Sensitivity estimation of metamaterial loaded planar waveguide, Opt Quant Electron, 47(7), 2015, $2277-2287$.

[35] S. A. Taya and Kh. Y. Elwasife, Field profile of asymmetric slab waveguide structure with LHM layers, J. Nano- Electron. Phys. 6(2), 2014, 5 .

[36] S. A. Taya, Dispersion properties of lossy, dispersive, and anisotropic left-handed material slab waveguide, Optik - Int. J. Light Electron Opt., 126(14), 2015, 1319-1323.

[37] S. A. Taya and D. M. Alamassi, Reflection and transmission from left-handed material structures using Lorentz and Drude medium models, Opto-Electron. Rev., 23(3), 2015, 214-221.

[38] S. A. Taya, A. A. Jarada and H. M. Kullab, Slab waveguide sensor utilizing left-handed material core and substrate layers, Optik Int. J. Light Electron Opt., 127(19), 2016, 7732-7739.

[39] T. Ergin, N. Stenger, P. Brenner, J.B. Pendry, and M. Wegener, Three-dimensional invisibility cloak at optical wavelengths, Science, J., 328(5976, 2010, 337-339.

[40] L.W. Li, Y.N. Li, T.S. Yeo, J.R. Mosig, and O.J. Martin, A broadband and high-gain metamaterial microstrip antenna, Appl. Phys. Lett. 96(16), 2010, 164-165.

[41] Zh. Zhang, Zh. Wang, L. Wang, Design principle of single- or double-layer wave-absorbers containing left-handed materials, Materials and Design, 30(9), 2009, 3908-3912.

[42] S. A. Taya and T. M. El-Agez, Comparing optical sensing using slab waveguides and total internal reflection ellipsometry, Turkish J. of Physics, 35(10), 2011, 31-36.

[43] T. M. El-Agez and S. A. Taya, Theoretical spectroscopic scan of the sensitivity of asymmetric slab waveguide sensors, Optica Applicata, 41(1), 2011, 89-95. 